\title{
Time series modeling of the cycle-to-cycle variability in h-BN based memristors
}

\author{
J. B. Roldán ${ }^{1}$, D. Maldonado ${ }^{1}$, F.J. Alonso², A. M. Roldán ${ }^{1}$, F. Hui ${ }^{3}$, Y. Shi ${ }^{4}$, F. Jiménez-Molinos ${ }^{1}$, \\ A.M. Aguilera ${ }^{2}$ and M. Lanza ${ }^{5}$
}

${ }^{1}$ Dep. Electrónica y Tecnología de Computadores. Universidad de Granada. Facultad de Ciencias, 18071, Granada, Spain

${ }^{2}$ Dep. Estadística e Investigación Operativa. Universidad de Granada. Facultad de Ciencias, 18071, Granada, Spain

${ }^{3}$ Faculty of Materials Science and Engineering, Technion - Israel Institute of Technology, Haifa 3200003, Israel

${ }^{4}$ IMEC, Kapeldreef 75, B-3001 Leuven, Belgium

${ }^{5}$ Physical Sciences and Engineering Division, King Abdullah University of Science and Technology (KAUST), Thuwal 23955-6900, Saudi Arabia

* Corresponding author Email: jroldan@ugr.es, mario.lanza@kaust.edu.sa

\begin{abstract}
We have characterized and modeled memristor devices based on the Au/Ti/multilayer h-BN/Au/Ti stack. Resistive switching (RS) operation has been analysed by extracting the reset and set voltages and currents. The evolution of the set and reset parameters along a RS series was mathematically modeled in a cycle-to-cycle (CTC) basis by means of the Time Series Analysis (TSA). To do so, the Autocorrelation Functions (ACF) and the Partial Autocorrelation Functions (PACF) have been calculated. These tools help to perform a comprehensive variability study and to obtain the corresponding analytical models within the TSA context. Finally, we have included this modeling procedure in a complete compact model such as the Stanford to be able to account for this variability at the circuit level. Experimental current versus voltage $(I-V)$ curves have been correctly fitted with the model.
\end{abstract}

Index Terms-- Memristor, variability, compact modeling, reliability, time series analysis, dielectric, two-dimensional material, electrical characterization.

\section{INTRODUCTION}

Memristors, predicted by Chua in 1971 [1], can be applied to different technologies [2]. Nowadays, there are different types of devices that can be considered as memristors; among them, there are the ones relying on ferroelectric, phase change or magnetic material properties [2-4]. Transition metal oxides have been extensively studied in this context [2-4]. In particular, resistive switching (RS) devices with filamentary charge conduction, based on $\mathrm{HfO}_{2}, \mathrm{TiO}_{2}, \mathrm{Al}_{2} \mathrm{O}_{3}$ dielectrics, among others, have shown outstanding features such as high endurance, good retention, low power consumption, CMOS technology compatibility, scalability, and capacity for being fabricated in 3D stacked structures [4-6].

Nevertheless, other dielectric alternatives based on 2D materials are being considered due to the outstanding features

We thank the Spanish Ministry of Science for project TEC201784321-C4-3-R, MTM2017-88708-P, PGC2018-098860-B-I00 and Junta de Andalucía for projects A-TIC-117-UGR18 and A-FQM345-UGR18 all with the support of the European Regional Development Fund. We also thank NSFC (61874075), MOST (BRICS2018-211-2DNEURO) and the Ministry of Finance of China (SX21400213). of the electron devices that employ them [7-10]. Intense research efforts are being conducted to describe yield, variability, reliability and stability in the field of $2 \mathrm{D}$ materials based solid-state nano/micro-electronic devices [9, 10]. 2D dielectric memristor technology can lead to solutions to some of the issues that show up in memristors industrial applications linked to non-volatile memories [2, 9], entropy sources for cryptographic hardware (random number generation and implementation of physical unclonable functions $[2,9,11])$ and, most important, neuromorphic computing $[2,8,10]$. In the latter case, the fabrication of devices that mimic the behavior of biological synapses is essential $[8,12,13]$. The capacity to build circuits to perform matrix-vector multiplications will allow the implementation of efficient hardware neural networks (HNN) that can greatly accelerate neuromorphic computing, and above all, reduce power consumption of brain-inspired designs and native artificial intelligence systems. In addition, properties such as spike timing dependent plasticity, long/short term plasticity, etc., facilitate the development of spiking neural networks that process information closely to what is done in the human brain [14].

For the use of these emerging technologies out of certain niche applications, both variability and implementation of mature simulation tools have to be addressed. We deal with both subjects in this work. It is known that the inherent random nature of the physical mechanisms behind RS makes memristor operation different to other type of electron devices $[2,5,6]$. In this respect, the device stochasticity has been studied and modeled from different viewpoints. For instance, advanced statistical distribution functions, e.g., Phase-type distributions have been successfully applied to deal with variability [15, 16]; approaches founded on functional data analysis were also used [17-19]; from another perspective, simulations linked to kinetic Monte Carlo techniques proved their adequacy [20, 21]. Here, we address CTC variability in h-BN memristors from the time series analysis perspective [22-24]. TSA has been proved to be an efficient tool for a comprehensive CTC variability characterization and modeling [22-24]. This modeling technique accounts for the "inertia" 
connected to RS processes; i.e., the dependencies of present set and reset events on previous RS operation is taken into consideration. This idea means keeping "modeling memory" of the RS previous operation; e.g., CF remnants left in past reset processes are considered when a new reset event is to be described. As far as we know, this is the first time this methodology has been used in 2D materials based devices.

\section{DEVICE FABRICATION AND MEASUREMENT}

The memristors we have fabricated consists of the following layers $(40 \mathrm{~nm} \mathrm{Au} / 10 \mathrm{~nm} \mathrm{Ti} /$ multilayer h-BN/40 $\mathrm{nm} \mathrm{Au} / 10 \mathrm{~nm} \mathrm{Ti} / 300 \mathrm{~nm} \mathrm{SiO} / 2 \mathrm{Si}$ ). E-beam evaporation was employed for the electrode deposition. The h-BN multilayer films were grown by chemical vapor deposition (CVD) on $\mathrm{Cu}$ foil and inserted between the electrodes via wet transfer. The $(I-V)$ curves were measured through a Karl Suss probe station connected to a semiconductor parameter analyzer under ramped voltage stress (RVS). Long RS series were obtained including complete and consecutive set and reset cycles. The voltage was applied to the top electrode, the bottom electrode was grounded.

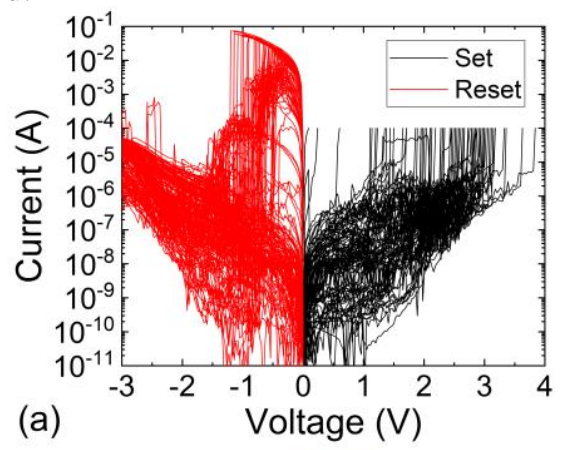

(b)

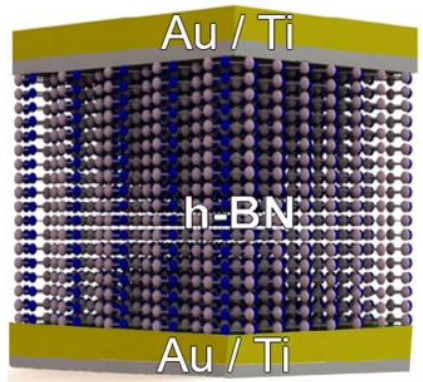

Figure 1. (a) Experimental current versus applied voltage for different RS cycles in a long series for the devices under study. 94 set (black curve) and reset (red curve) processes have been plotted. (b) Schematic of the fabricated devices, $\mathrm{I}_{\mathrm{CC}}=10^{-4} \mathrm{~A}$

The measured I-V curves are shown in Fig. 1 along with the device layer schema. The charge conduction is known to be filamentary, RS is produced by means of the rupture and creation of conductive filaments (CFs) that short the electrodes [2, 7]. The mechanisms involved in the CFs formation and rupture are random, this randomness is linked to the cycle-to-cycle (CTC) variability.

\section{TSA MODELING, RESULTS AND DISCUSSION}

We have obtained the set and reset voltages and currents by identifying the higher I-V slope point and the maximum current point respectively (Fig. 2).

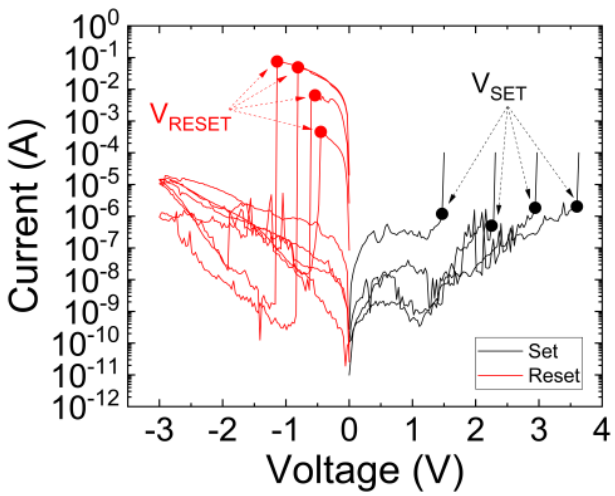

Figure 2. Experimental current versus applied voltage for different RS cycles. The points where $V_{\text {SET }}$ and $V_{\text {RESET }}$ are calculated have been marked. We have identified the higher I-V slope point for the set voltage determination and the maximum current point for the reset voltage.

The set and reset (absolute value) voltages cumulative distribution functions were plotted (Fig. 3) to describe the cycle-to-cycle variability for 94 RS cycles.

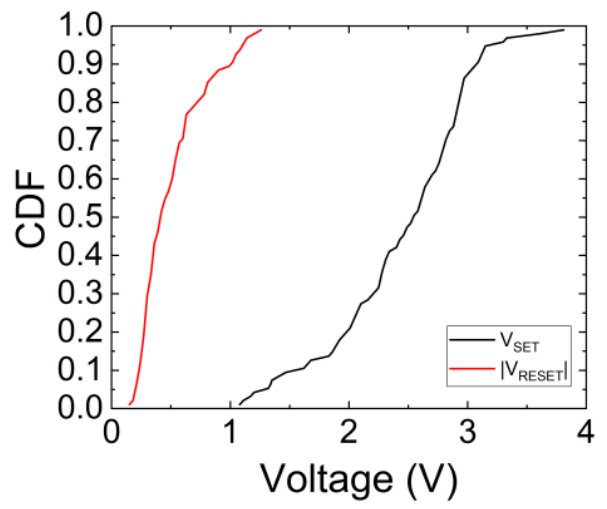

Figure 3. Cumulative Distribution Functions (CDF) for the calculated parameters $\mathrm{V}_{\mathrm{SET}}$ and $\left|\mathrm{V}_{\mathrm{RESET}}\right|$.

The corresponding ACF (Fig. 4) and PACF (Fig. 5) plots have been calculated [22-23]. A useful measure of the degree of dependence among the data (reset and set voltages) of different cycles is the autocorrelation function. The ACF (Eq. 1 ), a function of the number of cycles, $k$, measures the influence/connection between $\mathrm{V}_{\text {RESET }}$ values separated by $k$ cycles ( $k$ distant lags in the RS series), this also works out for the other RS parameters, such as $\mathrm{V}_{\mathrm{SET}}$ [22, 25-26]. For the $\mathrm{V}_{\text {SET }}$ (Fig. 4) we have several components, the corresponding to the previous cycle and two others that dominate over the reset. The values have to be compared with the threshold bounds, which depends on the number of cycles of the series $[25,26]$. For the $\mathrm{V}_{\text {RESET }}$ we have just one component. The $\mathrm{ACF}, \rho$, is given in Equation 1,

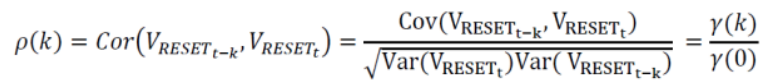

where Cov is the covariance, Var is the variance, $t$ is the actual $\mathrm{V}_{\mathrm{RESET}}$ value and $(t-k)$ the value of $k$ cycles before [25].

To better understand the ACF, we stress that it accounts for the "inertia" produced in the RS parameter values by the CF remnants remaining in each RS cycle (these remnants characterize the influence of previous RS cycles on the current one). The PACF describes the same correlation than ACF 
eliminating the dependency due to the intermediate lags (1, $2 \ldots, \mathrm{k}-1)[22,23]$.
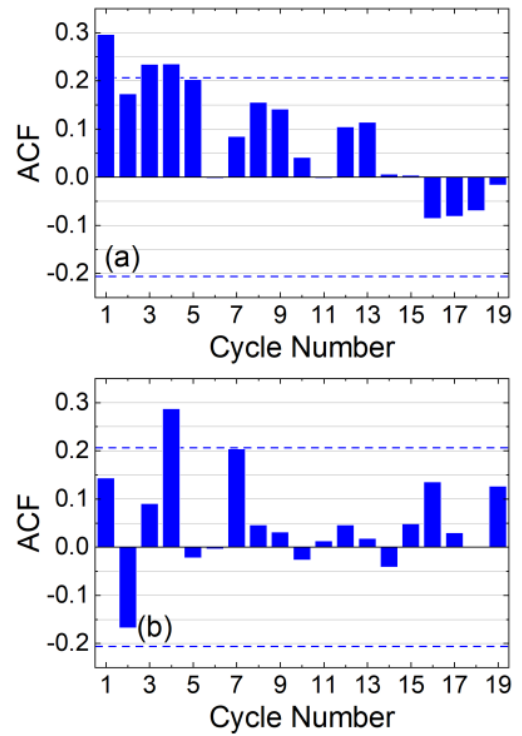

Figure 4. ACF vs cycle lag (distance apart in cycles within a RS series; for a cycle lag 1, the ACF of consecutive cycles is measured) for the (a) $V_{\text {SET }}$ and (b) $V_{\text {RESET }}$ series. The minimum and maximum threshold bounds are 0.20628 and -0.20628 respectively, shown by the dashed lines. These values depend on the number of data in the sample [22].
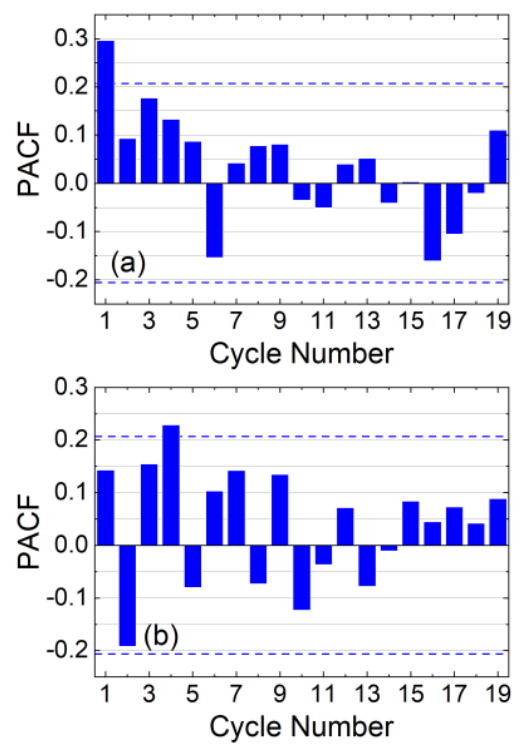

Figure 5. PACF vs cycle lag for the (a) $V_{\text {SET }}$ and (b) $V_{\text {RESET }}$ series described in Fig. 3. The minimum and maximum threshold bounds are 0.20628 and 0.20628 respectively, shown by the dashed lines.

The TSA techniques (along with the results of Figs. 4 and 5) allowed us to obtain the analytical expression of the time series for prediction of $\mathrm{V}_{\text {SET }}$ and $\mathrm{V}_{\text {RESET }}$, see Table I. For the determination of the constants included in the equations and the number of terms and the type of models, see ref. [22, 25]. An Auto Regressive AR (1) model was employed for $V_{\text {SET }}$ data, the $V_{\text {RESET }}$ model proposed, in this case for the absolute value, is an AR (4) model with null coefficients in cycles t-1, $\mathrm{t}-2$ and $\mathrm{t}-3$ :

\begin{tabular}{|c|}
\hline $\begin{array}{c}\text { TSA analytical expressions for prediction along a } \\
\text { RS series for } \mathbf{V}_{\text {SET }} \text { and } \mathbf{V}_{\text {RESET }}\end{array}$ \\
\hline Vset $_{t}=1.68+0.3153$ Vset $_{t-1}$ \\
\hline Vreset $_{t}=0.3536+0.3217$ Vreset $_{t-4}$ \\
\hline
\end{tabular}

Table I. Time series analytical expressions based on a first order $\left(\mathrm{V}_{\mathrm{SET}}\right)$ and fourth order $\left(\mathrm{V}_{\mathrm{RESET}}\right) \mathrm{TSA}$ autoregressive models.

See in Fig. 6 the measured and modeled values of the set and reset voltages, which were obtained by using the expressions of Table I. As can be seen, a reasonably good prediction can be performed for the cycle-to-cycle variability. This type of modeling, as far as we know, is the most accurate approach to deal with cycle-to-cycle variability because in addition to models where the $\mathrm{V}_{\text {RESET }}$ and $\mathrm{V}_{\text {SET }}$ variations are considered, the RS memory effects linked to resistive switching and past dependencies can be taken into account. It is also important to highlight that the time series is formulated accounting for the statistical features of the whole series; therefore, these characteristics are included in the corresponding analytical expression "on average". In this respect, the model reproduces the ups and downs (trend) of the experimental data; nevertheless, the sudden peaks in the data cannot be reproduced with a model that it's forged with the averaged features of the whole series. It is important to highlight that this sort of variability is important in the context of hardware neural networks, since although the training process could be affected by the variations in a conductance multilevel operation regime, variability could also compensate training weaknesses such as overfitting, when using memristors to implement synaptic devices.
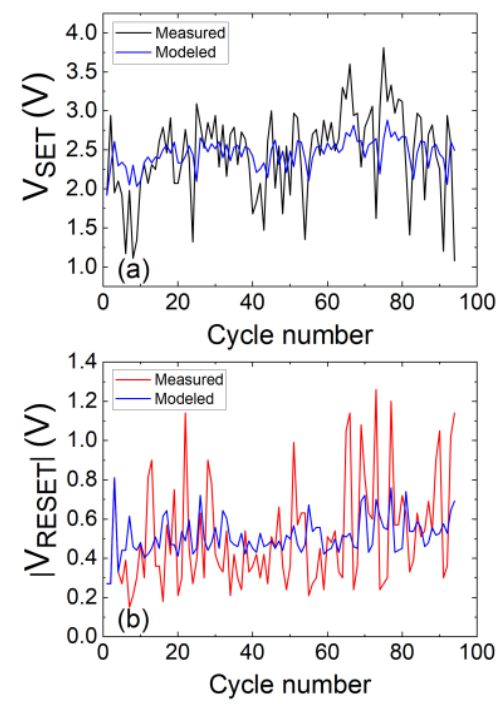

Figure 6. (a) $\mathrm{v}_{\mathrm{SET}}$ (b) $\left|\mathrm{v}_{\mathrm{RESET}}\right|$ versus cycle number for the RS series under consideration. 


\begin{tabular}{|c|c|c|c|}
\hline \multicolumn{4}{|c|}{ Stanford model parameters } \\
\hline Device Parameters & Unit & \multicolumn{2}{|c|}{ Resistive Switching } \\
\hline & & SET & RESET \\
\hline$V_{o}$ & $\mathrm{~V}$ & 0.75 & 0.45 \\
\hline$I_{0}$ & $\mathrm{~mA}$ & 0.2 & 18 \\
\hline$g_{0}$ & $\mathrm{~nm}$ & 0.2 & 0.143 \\
\hline$v_{0}$ & $\mathrm{~m} / \mathrm{s}$ & \multicolumn{3}{|c|}{$5 \times 10^{6}$} \\
\hline$\alpha$ & - & \multicolumn{3}{|c|}{} \\
\hline$\beta$ & - & 1 & 28 \\
\hline$\gamma_{0}$ & - & 16 & 44 \\
\hline
\end{tabular}

Table II. Stanford model parameters employed for the experimental device under study.

Finally, a simulation based on the Stanford model [27-28] has been implemented (see the results in Fig. 7). A reasonably good fit of the experimental curves was obtained using different sets of parameters for the set and reset curves, as it was proposed in ref. [28], (see Fig. 7a). This fact shows the flexibility of the model, it works reasonably well although the nature of the dielectric could lead to complex charge transport mechanisms to explain the device operation. We have included the time series formula (Table I) and we were able to account for the experimental cycle-to-cycle variability we measured (Figs. 3, 6 and 7b) under the needed transformations to describe the resistive switching parameters variability in terms of the internal Stanford model parameters. This modeling was devoted to the reset and set voltages; nevertheless, other effects of the stochasticity inherent to resistive switching, such as different conductive filaments geometries, take place in each cycle. Therefore, other features (current levels, I-V curve shapes...) would have to be considered from the modeling viewpoint in the future.
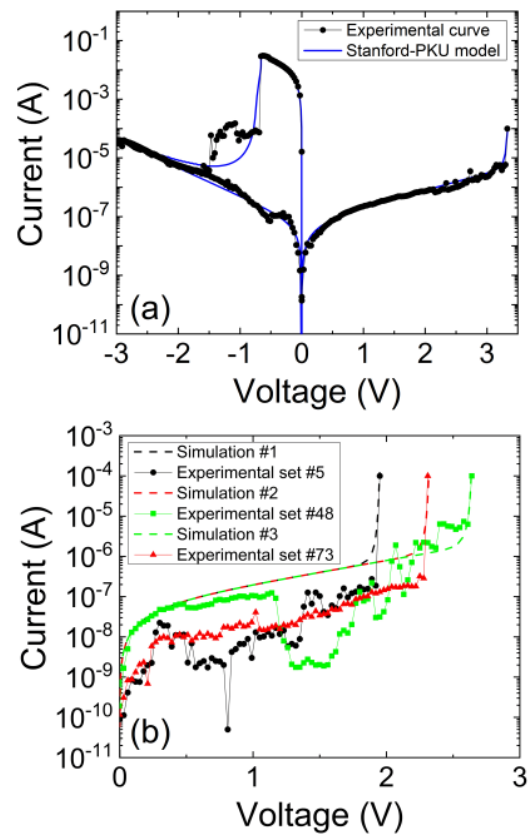

Figure 7. (a) Experimental cycle (black symbols) vs. voltage for the device measured. Modeled data employing the Stanford model (blue line). (b) Simulations as result of implementing the TSA expression of $\mathrm{V}_{\text {SET }}$ on the Stanford model (dashed lines) and experimental cycles (symbols).

\section{CONCLUSIONS}

We have measured and modeled memristors fabricated with the $\mathrm{Au} / \mathrm{Ti} / \mathrm{h}-\mathrm{BN} / \mathrm{Au} / \mathrm{Ti}$ stack. Resistive switching (RS) operation has been analyzed; in particular, the evolution of the set and reset parameters along a RS series. Time series analysis techniques were employed to obtain analytical expressions to describe cycle-to-cycle variability. The equations obtained have been incorporated in the Stanford model to correctly reproduce the experimental measurements.

\section{REFERENCES}

[1] L. Chua, "Memristor-The missing circuit element", in IEEE Transactions on Circuit Theory, vol. 18, no. 5, pp. 507-519, 1971.

[2] M. Lanza, et al., "Recommended methods to study resistive switching devices", Advanced Electronics Materials, 5, 1800143, 2019.

[3] M.A. Villena, J.B. Roldán, F. Jiménez-Molinos, E. Miranda, J. Suñé, M. Lanza, "SIM2RRAM: A physical model for RRAM devices simulation", Journal of Computational Electronics, 16, pp. 1095-1120, 2017.

[4] F. Pan, S. Gao, C. Chen, C. Song, F. Zeng, "Recent progress in resistive random access memories: materials, switching mechanisms and performance", Materials Science and Engineering, 83, pp. 1-59, 2014.

[5] D. Ielmini, "Modeling the universal set/reset characteristics of bipolar RRAM by field-and temperature-driven filament growth", IEEE Transactions on Electron Devices, 58(12), pp. 4309-4317, 2015.

[6] D. Ielmini, R. Waser. "Resistive Switching: From Fundamentals of Nanoionic Redox Processes to Memristive Device Applications", Wiley-VCH, 2015.

[7] F. Hui, M. A. Villena, W. Fang, A.-Y. Lu, J. Kong, Y. Shi, X. Jing, K. Zhu, M. Lanza, "Synthesis of large-area multilayer hexagonal boron nitride sheets on iron substrates and its use in resistive switching devices”, 2D Materials, 5, 031011, 2018.

[8] Y. Shi, X. Liang, B. Yuan, V. Chen, H. Li, F. Hui, Z. Yu, F. Yuan, E. Pop, H.-S. P. Wong, M. Lanza "Electronic synapses made of layered two-dimensional materials", Nature Electronics 1, 458-465, 2018.

[9] Y. Illarionov, T. Knobloch, M. Lanza, D. Akinwande, M.I. Vexler, T. Mueller, M. Lemme, G. Fiori, F. Schwierz, and T. Grasser, "Insulators for 2D Nanoelectronics", Nature Communications, 11, 3385, 2020.

[10] M. Lanza, Q. Smets, C. Huyghebaert, L.-J. Li, "Yield, variability, reliability, and stability of two-dimensional materials based solid-state electronic devices", Nature Communications, 11, 5689, 2020.

[11] F.M. Puglisi, N. Zagni, L. Larcher, P. Pavan, "Random Telegraph Noise in Resistive Random Access Memories: Compact Modeling and Advanced Circuit Design", IEEE Transactions on Electron Devices, 65, pp. $2964-2972,2018$.

[12] M. Zidan, J.P. Strachan, W. Lu, "The future of electronics based on memristive systems", Nature Electronics, 1, 22-29, 2018.

[13] S. Yu, Y. Wu, R. Jeyasingh, D. Kuzum, H.-S. Wong, "An electronic synapse device based on metal oxide resistive switching memory for neuromorphic computation", IEEE Transactions on Electron Devices, 58 (8), pp. 2729-2737, 2011. doi: 10.1109/TED.2011.2147791.

[14] N. Zheng, P. Mazumder, "Learning in Energy-Efficient Neuromorphic Computing: Algorithm and Architecture Co-Design", Wiley, 2019.

[15] C. Acal, J.E. Ruiz-Castro, A. M. Aguilera, F. Jiménez-Molinos, J.B. Roldán, "Phase-type distributions for studying variability in resistive memories", Journal of Computational and Applied Mathematics, 345, pp. 23-32, 2019.

[16] E. Pérez, D. Maldonado, C. Acal, J.E. Ruiz-Castro, F.J. Alonso, A.M. Aguilera, F. Jiménez-Molinos, Ch. Wenger, J.B. Roldán, "Analysis of the statistics of device-to-device and cycle-to-cycle variability in TiN/Ti/Al:HfO $/$ /TiN RRAMs", Microelectronics Engineering, 214, pp. 104-109, 2019.

[17] M. C. Aguilera-Morillo, A. M. Aguilera, F. Jiménez-Molinos, J.B. Roldán, "Stochastic Modelling of Random Access Memories Reset Transitions", Mathematics and Computers in Simulation, 159 pp. 197209, 2019. 
[18] A.M. Aguilera, C. Acal, M.C. Aguilera-Morillo, F. Jiménez-Molinos, J.B. Roldán, "Homogeneity problem for basis expansion of functional data with applications to resistive memories", Mathematics and Computers in Simulation, in press, 2021.

[19] J.E. Ruiz-Castro, C. Acal, A.M. Aguilera, M.C. Aguilera-Morillo, J.B. Roldán, "Linear-Phase-Type probability modelling of functional PCA with applications to resistive memories", Mathematics and Computers in Simulation, in press, 2021.

[20] S. Aldana, P. García-Fernández, R. Romero-Zaliz, M.B. González, F. Jiménez-Molinos, F. Gómez-Campos, F. Campabadal, J.B. Roldán, "Resistive Switching in $\mathrm{HfO} 2$ based valence change memories, a comprehensive 3D kinetic Monte Carlo approach", Journal of Physics D: Applied Physics, 53, 225106, 2020.

[21] A. Padovani, L. Larcher, O. Pirrotta, L. Vandelli, G. Bersuker, "Microscopic Modeling of HfOx RRAM Operations: From Forming to Switching", IEEE Transactions on Electron Devices, 62(6), pp. 19982006, 2015.

[22] J. B. Roldán, F. J. Alonso, A. M. Aguilera, D. Maldonado, M. Lanza, "Time series statistical analysis: a powerful tool to evaluate the variability of resistive switching memories", Journal of Applied Physics, 125, 174504, 2019.
[23] F.J. Alonso, D. Maldonado, A.M. Aguilera, J. B. Roldan, "Memristor variability and stochastic physical properties modeling from a multivariate time series approach", Chaos, Solitons \& Fractals, 143, 110461, 2021.

[24] N. Rodriguez, D. Maldonado, F.J. Romero, F.J. Alonso, A.M. Aguilera, A. Godoy, F. Jimenez-Molinos, F.G. Ruiz, J.B. Roldan, "Resistive switching and charge transport in laser-fabricated graphene oxide memristors: a Time Series and Quantum Point Contact modelling approach", Materials, 12, 3734, 2019.

[25] P. J. Brockwell, R. A. Davis, "Introduction to Time Series and Forecasting", Second Edition, Springer, 2002.

[26] S.Bisgaard, M.Kulahci, "Time series analysis and forecasting by example", Wiley, 2011.

[27] X. Guan, S. Yu, and H-S.P Wong, "A SPICE Compact Model of Metal Oxide Resistive Switching Memory with Variations", IEEE Elec. Dev. Lett., vol. 33, pp. 1405-1407, 2012.

[28] Z. Jiang, Y. Wu, S. Yu, L. Yang, K. Song, Z. Karim, H.-S. P. Wong, "A Compact Model for Metal-Oxide Resistive Random Access Memory With Experiment Verification", IEEE Transactions on Electron Devices, vol. 63, no. 5, pp. 1884-1892, May 2016. 